\title{
Extracapsular lymph node involvement in patients with esophageal cancer treated with neoadjuvant chemoradiation therapy followed by surgery: the closer you look, the less you see
}

\author{
Yin-Kai Chao \\ Division of Thoracic and Cardiovascular Surgery, Chang Gung Memorial Hospital at Linkou, College of Medicine, Chang Gung University, \\ Taoyuan, Taiwan \\ Correspondence to: Yin-Kai Chao. Division of Thoracic and Cardiovascular Surgery, Chang Gung Memorial Hospital at Linkou, Chang Gung \\ University, 5, Fushing Street, Gueishan Shiang, Taoyuan, Taiwan. Email: chaoyk@cgmh.org.tw. \\ Provenance: This is an Invited Editorial commissioned by Section Editor Dr. Wankun Chen (Department of Anesthesiology, Fudan University \\ Shanghai Cancer Center, Shanghai, China). \\ Comment on: Depypere LP, Moons J, Mariette C, et al. Impact of extracapsular lymph node involvement after neoadjuvant chemoradiation therapy \\ followed by surgery in carcinoma of the esophagus: a multicenter study. Ann Surg 2017. [Epub ahead of print].
}

Submitted Sep 30, 2017. Accepted for publication Oct 12, 2017.

doi: $10.21037 /$ jtd.2017.10.53

View this article at: http://dx.doi.org/10.21037/jtd.2017.10.53

Esophageal carcinoma is a highly aggressive malignancy that generally portends a poor prognosis (1). Despite significant advances in surgical techniques, recurrences after resection remain common. The presence of lymph node metastases is widely recognized as the most important predictor of disease recurrence (2). Besides the presence of extra- or intracapsular involvement, the number and location of metastatic lymph nodes as well as the metastatic lymph node ratio may help to further improve prognostication and refine individual patient risk $(3,4)$. Notably, the number of lymph node metastases is an essential $\mathrm{N}$-staging criterion in the seventh edition of the American Joint Committee on Cancer (AJCC) staging system (5); however, the status of capsular invasion has not yet been included.

Extracapsular lymph node involvement (ELNI) is traditionally defined as the extension of cancer cells through the nodal capsule into the perinodal fatty tissue (6). The ability of tumor cells to spread not only into a lymph node but also to penetrate the lymph node capsule within an immunologically hostile environment reflects the primary tumor cell's migratory and invasive capacity, being ultimately an indicator of its biological aggressiveness (7). In this context, the "metastases-to-metastases" theory proposes that extracapsular metastatic cells could promote the formation of novel foci, ultimately facilitating systemic dissemination (2).
ELNI is an adverse predictor of survival in patients with esophageal cancer, independent of tumor histology [i.e., adenocarcinoma (ADC) versus squamous cell carcinoma (SCC)] (8-10). Although the same concept could theoretically be applied to patients with locally advanced esophageal cancer treated with neoadjuvant chemoradiotherapy (nCRT), direct evidence in support of this possibility remains scarce. In this regard, the few published studies focusing on the prognostic significance of ELNI in esophageal cancer included a variable number of patients treated with nCRT followed by surgery; in addition, they yielded conflicting results (11-13).

The recent study by Depypere and colleagues should be commended for filling the knowledge gap on the prognostic significance of ELNI in nCRT-treated patients with esophageal cancer (14). The authors' results indicated that the prognostic impact of ELNI was dependent on the histological subtype (14). Specifically, ELNI was an independent adverse prognostic factor for survival in patients with esophageal SCC, but not in those with ADC. Moreover, the presence of ELNI was the strongest predictor of poor overall survival in SCC patients, being the variable showing the highest hazard ratio $(2.29,95 \%$ confidence interval: 1.52-3.47).

Intriguingly, subgroup analysis revealed that the detrimental prognostic significance of ELNI in SCC was 
limited to patients who had less than two nodal metastases (i.e., ypN1). In contrast, the presence or absence of ELNI did not show any survival impact for patients classified in the ypN2 and ypN3 categories. The main strength of the study lies in its large sample size $(>1,500$ patients enrolled in six high-volume European centers) that provides a high statistical power to support the prognostic role of ELNI in nCRT-treated patients with esophageal cancer.

The question of how these results can influence our clinical practice merits comment. For the first time, the upcoming eighth edition of the AJCC staging system will stage patients according to the use of preoperative therapy (yes vs. no) (15). However, post-treatment ypN1 will be solely staged as stage IIIA disease, regardless of both ypT stage and the capsular invasion status. In contrast, it should be emphasized that patients staged as having ypN1 disease are a heterogeneous group. The results of Depypere and coworkers clearly indicate that patients with ypN1 could at least be divided into subgroups based on the presence or absence of ELNI (14). Specifically, the prognosis of ypN1 in the absence of ELNI was as favorable as that observed in ypN0.

However, caution should be exercised when the prognostic impact of ELNI is translated into clinical practice. First, ELNI is not uniformly defined in the published literature. For example, differently from previously published papers (11-13), the study by Depypere et al. did not classify tumor deposits (TDs) in the periesophageal fat as ELNI when a recognizable lymph node structure was absent in the nearby (14). The reason motivating this choice is unclear. The prognostic significance of TDs has been assessed in several studies focusing on gastrointestinal malignancies, especially colorectal cancer. The results indicated that the presence of TDs has an adverse prognostic impact regardless the use of neoadjuvant CRT or not $(16,17)$. In addition, TDs are already considered as a form of ELNI and classified as N1c disease in the seventh edition of the AJCC staging system (18). Second, nCRT can "sterilize" some lymph nodes initially showing metastatic involvement. "Sterilization"—-defined as pathological evidence of non-viable metastatic tumor cells in the affected node-is associated with poorer outcomes as compared with truly negative lymph nodes (19). The current practice of considering as pathologically negative lymph nodes showing areas of tumor necrosis but lacking viable cancer cells does probably result in understaging. However, differently from the well-accepted use of the tumor regression grade for pathological grading of primary tumor regression (20), the staging of "sterilized" nodes was not specifically addressed in the study (21). Third, the potential prognostic significance of the number of lymph nodes showing ELNI (which was shown to predict outcomes in other malignancies) was not reported. All of these important research questions should be addressed in future studies.

In conclusion, the type of lymph node involvement (intracapsular versus extracapsular) provides valuable prognostic information for patients with esophageal SCC treated with nCRT followed by surgery, especially in presence of limited nodal disease burden (i.e., ypN1). Besides the number of metastatic nodes, we suggest that both the presence and the number of nodes showing ELNI should routinely be included in pathological reports of patients with esophageal SCC.

\section{Acknowledgements}

Funding: This work was supported by grant CORPG3G0771 from the Chang Gung Memorial Hospital, Taiwan.

\section{Footnote}

Conflicts of Interest: The author has no conflicts of interest to declare.

\section{References}

1. Enzinger PC, Mayer RJ. Esophageal cancer. N Engl J Med 2003;349:2241-52.

2. Kayani B, Zacharakis E, Ahmed K, et al. Lymph node metastases and prognosis in oesophageal carcinoma-a systematic review. Eur J Surg Oncol 2011;37:747-53.

3. Mariette C, Piessen G, Briez N, et al. The number of metastatic lymph nodes and the ratio between metastatic and examined lymph nodes are independent prognostic factors in esophageal cancer regardless of neoadjuvant chemoradiation or lymphadenectomy extent. Ann Surg 2008;247:365-71.

4. Anderegg MC, Lagarde SM, Jagadesham VP, et al. Prognostic significance of the location of lymph node metastases in patients with adenocarcinoma of the distal esophagus or gastroesophageal junction. Ann Surg 2016;264:847-53.

5. Rice TW, Blackstone EH, Rusch VW. 7th edition of the AJCC Cancer Staging Manual: esophagus and esophagogastric junction. Ann Surg Oncol 2010;17:1721-4.

6. Wind J, Lagarde SM, ten Kate FJ, et al. A systematic review on the significance of extracapsular lymph node involvement in gastrointestinal malignancies. Eur J Surg 
Oncol 2007;33:401-8.

7. Lee WY, Shin DY, Kim HJ, et al. Prognostic significance of epithelial-mesenchymal transition of extracapsular spread tumors in lymph node metastases of head and neck cancer. Annals of surgical oncology. 2014;21:1904-11.

8. Sakai M, Suzuki S, Sano A, et al. Significance of lymph node capsular invasion in esophageal squamous cell carcinoma. Ann Surg Oncol 2012;19:1911-7.

9. Lagarde SM, Fiebo J, de Boer DJ, et al. Extracapsular lymph node involvement in node-positive patients with adenocarcinoma of the distal esophagus or gastroesophageal junction. Am J Surg Pathol 2006;30:171-6.

10. Lerut T, Coosemans W, Decker G, et al. Extracapsular lymph node involvement is a negative prognostic factor in T3 adenocarcinoma of the distal esophagus and gastroesophageal junction. J Thorac Cardiovasc Surg 2003;126:1121-8.

11. D'Annoville T, D'Journo XB, Loundou A, et al. Prognostic impact of the extracapsular lymph node involvement on disease-free survival according to the 7 th edition of American Joint Committee on Cancer Staging System. Eur J Cardiothorac Surg 2013;44:e207-11.

12. D'Journo XB, Avaro JP, Michelet P, et al. Extracapsular lymph node involvement is a negative prognostic factor after neoadjuvant chemoradiotherapy in locally advanced esophageal cancer. J Thorac Oncol 2009;4:534-9.

13. Metzger R, Bollschweiler E, Drebber U, et al. Neoadjuvant chemoradiotherapy for esophageal cancer: impact on extracapsular lymph node involvement. World J Gastroenterol 2010;16:1986-92.

14. Depypere LP, Moons J, Mariette C, et al. Impact of

Cite this article as: Chao YK. Extracapsular lymph node involvement in patients with esophageal cancer treated with neoadjuvant chemoradiation therapy followed by surgery: The closer you look, the less you see. J Thorac Dis 2017;9(11):42704272. doi: $10.21037 /$ jtd.2017.10.53 extracapsular lymph node involvement after neoadjuvant chemoradiation therapy followed by surgery in carcinoma of the esophagus: a multicenter study. Ann Surg 2017. [Epub ahead of print].

15. Rice TW, Gress DM, Patil DT, et al. Cancer of the esophagus and esophagogastric junction: Major changes in the American Joint Committee on Cancer eighth edition cancer staging manual. CA Cancer J Clin 2017;67:304-17.

16. Gopal P, Lu P, Ayers GD, et al. Tumor deposits in rectal adenocarcinoma after neoadjuvant chemoradiation are associated with poor prognosis. Mod Pathol 2014;27:1281-7.

17. Song JS, Chang HJ, Kim DY, et al. Is the N1c category of the new American Joint Committee on cancer staging system applicable to patients with rectal cancer who receive preoperative chemoradiotherapy? Cancer 2011;117:3917-24.

18. Jin M, Roth R, Rock JB, et al. The impact of tumor deposits on colonic adenocarcinoma AJCC TNM staging and outcome. Am J Surg Pathol 2015;39:109-15.

19. Nieman DR, Peyre CG, Watson TJ, et al. Neoadjuvant treatment response in negative nodes is an important prognosticator after esophagectomy. Ann Thorac Surg 2015;99:277-83.

20. Mandard AM, Dalibard F, Mandard JC, et al. Pathologic assessment of tumor regression after preoperative chemoradiotherapy of esophageal carcinoma. Clinicopathologic correlations. Cancer 1994;73:2680-6.

21. Bollschweiler E, Hölscher AH, Metzger R, et al. Prognostic significance of a new grading system of lymph node morphology after neoadjuvant radiochemotherapy for esophageal cancer. Ann Thorac Surg 2011;92:2020-7. 